\title{
LAURENCE STERNES BILDBENUTZUNG*
}

Laurence Sterne zitiert in Tristram Shandy und der Sentimental Journey in größter Breite, in den meisten Fällen ohne die Herkunft seiner Quellen anzugeben. Er ruft die gesamte satirische Tradition auf, der er sich unmittelbar verbunden sieht: Rabelais, Cervantes, Paul Scarron, Pope und Swift. Um die zeitgenössischen Novellenschreiber, auch die satirisch verfahrenden wie Fielding oder Goldsmith, macht er verblüffenderweise einen Bogen, offenbar will er mit ihnen nicht in einen Topf geworfen werden. Das scheint seiner Erzählhaltung geschuldet, die das kontinuierliche Fortschreiten der Geschichte wie die Pest vermeidet, vielmehr primär von den Digressionen, von den Abweichungen, lebt. Sein Verfahren bricht mit allen literarischen Traditionen, somit ist auch eine gattungsmäßige Zuordnung seiner Texte nicht eigentlich möglich. Viel ist um diese Frage gestritten worden, sie ist nicht wirklich zielführend. Wäre die Gattung benennbar, so wäre auch der Sinn des Erzählten fixierbar, und ebendies scheut Sterne wie der Teufel das Weihwasser. Seine literarische Form bleibt offen, verschiedene literarische Prinzipien werden miteinander verschränkt, ohne dass sich daraus eine geschlossene Form ergäbe.

Das satirische Prinzip ist sicherlich das wichtigste, es steht - und nur dies soll hier verfolgt werden - in engstem Zusammenhang mit der Zitatpraxis. Nun erschöpft sich Sternes Zitatverfahren mitnichten in der Ausbeutung der satirischen Tradition. Von besonderer Wichtigkeit ist Robert Burtons Melancholietraktat The Anatomy of Melancholy von 1621, nicht nur weil Melancholie geradezu das natürliche Korrelat zu einer satirischen Grundhaltung ist, sondern vor allem weil Burton die Zitatpraxis selbst reflektiert und in seinem eigenen Text nach dem Prinzip der Zitatcollage verfährt, im Wissen darum, dass das Zitierte im neuen Zusammenhang einer Sinnverschiebung unterliegt, ein neuer Text entsteht, andererseits aber alles unter der Sonne schon einmal gedacht wurde. Nun ist das Verblüffende, dass alles Zitierte bei Sterne in gebrochener Form erscheint, auch dasjenige, dessen Mitteilung er im Prinzip unterschreiben könnte, ja müsste, wie die pragmatische „Charity“-Moral der berühmten latitudinarischen Predigtbischöfe vom späten 17. Jahrhundert bis zu Sternes Gegenwart, von Tillotson oder Clarke bis zu Herring oder Hoadly.'

* Der vorliegende Text entspricht zu großen Teilen dem Kapitel „Laurence Sterne und van Dyck" in meinem neuen Buch Great wits jump. Laurence Sterne und die bildende Kunst, das 2011 im Fink Verlag erschienen ist.

$\mathrm{Zu}$ Sterne und „latitudinarianism“ siehe Ian Campbell Ross, Laurence Sterne. A Life, Oxford, 2002, Index; Manfred Pfister, Laurence Sterne, Horndon, 2001, S. 23-25; Werner Busch, Das 
Denn die Brüche entstehen nicht nur durch das Versetzen der Textbausteine in neue Zusammenhänge - theoretisch könnten sie hier ihre ursprüngliche Aussagekraft durchaus bewahren, es kommt auf den neuen Kontext an -, vielmehr resultieren sie aus dem grundsätzlichen Zweifel an der Möglichkeit von Eindeutigkeit einer jeden Aussage, ja, von Verbindlichkeit und definierter Wortbedeutung überhaupt. Kein Wunder, dass Dr. Johnson, der Schöpfer des englischen Wörterbuches, Sterne nicht mochte. ${ }^{2}$ Nichts ist im 18. Jahrhundert mehr diskutiert worden als das sich aus dieser Einsicht ergebende Rezeptionsund Kommunikationsproblem, gerade auch auf ästhetischem Gebiet: „,E]ach mind perceives a different beauty. “3

Und auch von daher ist neben den Satirikern John Locke mit seinem Essay Concerning Human Understanding von 1690 eine von Sternes wichtigsten Quellen. Vieles, was Locke zur Bildung von Bewusstsein auf der Basis assoziativer Gedankenverbindungen beobachtet, kann Sterne unterschreiben, die daraus bei Locke gezogenen Konsequenzen jedoch mitnichten. Er glaubt schlicht nicht an die Möglichkeit einer konsequenten logischen Befolgung von einmal als richtig Erkanntem, an die Möglichkeit der systematischen Ausschaltung von unlogischen Gedankenverbindungen, vielmehr sieht er das Leben gerade davon geprägt. Für ihn gibt es keine fixen Bedeutungen, sondern nur fixe Ideen, die sich zu Obsessionen auswachsen können. Sie mögen absurde, komische, pathologische Züge annehmen, gänzlich negativ zu werten sind allerdings auch sie nicht, da sie der Natur des Menschen entsprechen.

Die „Hobby-Horses“, die Walter und Onkel Toby, die Protagonisten von Tristram Shandy, vom Morgen bis zum Abend reiten und die, da sie unterschiedliche, sich im Prinzip ausschließende Referenzräume aufweisen - und zwar Verstand und Gefühl -, notwendig zu Kommunikationsstörungen führen, können nicht verhindern, dass die beiden sich herzlich zugetan sind, ja, den anderen mit seinen auf Missverständnissen beruhenden Reaktionen brauchen, um dem eigenen Steckenpferd diese oder jene Richtung geben zu können, sie können nur galoppieren, indem sie sich vergaloppieren. Dieses sonderbare Verfahren ähnelt entschieden dem Prinzip der Digression. Die Abschweifung vom Gang der Dinge, den der schreibende Sterne ohnedies nicht vorab weiß, bringt den Abschweifenden bzw. den Leser der Abschweifung verwandelt zum Ausgangspunkt zurück, selbst wenn die Abschweifung so gut wie nichts mit dem (vermeintlichen) Hauptweg der Geschichte zu tun hat und nur durch eine Assoziation, die ganz anderen Erfahrungszusammenhängen entstammen

sentimentalische Bild. Die Krise der Kunst im 18. Jahrhundert und die Geburt der Moderne, München, 1993, S. 26-30.

2 James Boswell, Life of Johnson, hrsg. v. Robert W. Chapman, überarb. v. J. D. Fleeman, Oxford, 1970, S. 696; Johnsonian Miscellanies, 2 Bde., hrsg. v. George Birkbeck Hill, Oxford, 1897, Bd. 2, S. 320 (Anecdotes of George Steevens).

David Hume, „Philosophical Essays Concerning Human Understanding [1748]“, in: ders., Essays Moral, Political, and Literary (The Works of David Hume, Bd. 1), London/Edinburgh u. a., 1904, S. 234 f. 
mag, ausgelöst wurde. Die Sache hat eine andere Färbung bekommen, aufgrund derer der Fortgang der Dinge in eine neue Richtung angestoßen werden kann; nur um durch eine neue Digression erneut verändert zu werden. ${ }^{4}$ So mäandriert das Geschehen. Was der Einzelne tun kann, ist, seinen subjektiven Eindrücken, die bei Sterne sehr häufig Seheindrücke sind, ihr Recht zu geben, im Wissen darum, dass diese Eindrücke dem Leben Färbung und vor allem Bewegung geben. Für den kranken Sterne ist Bewegung Leben, Stillstand Tod, wie er selbst im siebten Buch von Tristram Shandy ausgesprochen hat: „Ich aber (der ich mager bin) denke anders; dass nämlich viel Motion ebensoviel Leben und ebensoviel Freude bedeutet - und stillstehen oder nur langsam vorwärtskommen Tod und Teufel -“.

Die Frage, die sich nun erhebt, lautet: Wie verarbeitet Sterne im einzelnen Seh- und Leseeindrücke? Bringt er Marker an, um dem Leser deutlich zu machen, dass hier Angeeignetes verarbeitet wird? Haben die Verfahren der Aneignung eine Tradition, an die Sterne anknüpfen kann, die er paraphrasieren, modifizieren oder karikieren kann? Dies sei an einem einzigen Beispiel untersucht, das der Forschung aus verschiedenen Gründen bisher entgangen ist, an dem sich jedoch die Sterne'schen Verfahren geradezu paradigmatisch und auf die Hauptgegenstände der gesamten Geschichte von Tristram Shandy bezogen erhellen lassen.

Die Zitatpraxis in der bildenden Kunst ist nicht etwa ein Produkt des über Rezeptionsfragen räsonierenden 18. Jahrhunderts, sondern klassisch-akademischer Brauch und empfohlene Kunstpraxis. Gelungene klassische Form- und Figurfindungen gewinnen normative Gültigkeit, ihre modifizierende Wiederverwendung belegt zweierlei: einerseits die Anknüpfung an ein bestimmtes verbindliches Anspruchsniveau bzw. seine Fortschreibung, die die Zugehörigkeit zu dieser Tradition legitimiert, und andererseits den Ausweis künstlerischer Innovationskraft. Erfindung bedeutet in diesem Zusammenhang Anverwandlung, aber auch Verfügbarmachen für neue Bedeutungshorizonte. Nicht selten verbirgt sich dahinter auch eine Gattungsreflexion bzw. eine Reflexion über angemessene Stilhöhen. Doch Sterne gibt dem Zitatverfahren einen völlig neuen Sinn.

Im dritten Buch von Tristram Shandy findet sich eine längere Passage, deren Bildhaftigkeit die Forschung immer betont hat ${ }^{6}$, sie stellt die vielleicht ausführlichste und eindringlichste Beschreibung einer Pose des gesamten Sterne'schen Textes dar, zumal sie über gleich mehrere Kapitel des dritten und vierten Buches verteilt ist, die beide noch 1760 erschienen. Man muss die

4 Sterne selbst zu den Digressionen in Tristram Shandy: 14. Kap., 1. Buch; 22. Kap., 1. Buch (Laurence Sterne, Leben und Ansichten von Tristram Shandy, Gentleman, übers. u. komm. v. Michael Walter, Frankfurt am Main, 2010 [zuerst 2006], S. 45-47 und 81-83).

Ebd., S. 522 (13. Kap., 7. Buch).

6 Am nachdrücklichsten betont von R. F. Brissenden, „Sterne and Painting“, in: Of Books and Humankind. Essays and Poems Presented to Bonamy Dobrée, hrsg. v. John Butt, London, 1964, S. 95, ohne jedoch irgendwelche Konsequenzen daraus zu ziehen. 
Teile wirklich zusammen sehen, denn die Passage ist nicht nur von einzelnen Kapiteln unterbrochen, sondern schließt die längste Digression des Textes überhaupt ein, die höchst anrüchige Geschichte von Slawkenbergius, die, ohne Kapitelangabe, dem vierten Buch vorgeschaltet ist. ${ }^{7}$ Wie alle Digressionen so soll auch diese in einer veränderten Einstellung zur auf der Stelle tretenden Geschichte zurückführen und damit auch der Geschichte eine zusätzliche subkutane, aber vielleicht damit die eigentliche Bedeutung geben. Die Geschichte von Slawkenbergius ist eine reine Nasengeschichte. Slawkenbergius verfügt über die längste denkbare Nase, und alle Frauen, einschließlich der Insassinnen eines ganzen Frauenklosters, sind verrückt nach ihr, während die Männer aufs Höchste verunsichert sind und die Lebendigkeit dieser Nase auf jede nur denkbare Weise in Frage zu stellen suchen, was bei den Frauen allerdings überhaupt nichts fruchtet. Und Sterne kann so sehr betonen, wie er möchte, dass, wenn er von Nasen redet, er auch nur Nasen und nichts anderes meint ${ }^{8}$; schon die mehrfache Betonung dieses Faktums macht deutlich, dass die Nase für das männliche Geschlecht steht und Slawkenbergius' Überpotenz gegen die Impotenz der betroffenen Männer, die nicht über eine solche Nase verfügen. Dies im Bewusstsein bekommen Tristrams Geburtsvorgang, von dem die Digression ihren Ausgang nahm, und Vater Walters anschließende Verzweiflung geradezu notwendig eine andere Färbung.

Walter und Toby sitzen im Parterre, während oben Dr. Slop mit seiner neuen Geburtszange Tristram, der noch nicht so heißt, ins Leben befördert. Doch die Zange zerdrückt ihm die Nase, Dr. Slop versucht aus Leinwand und Fischbein aus dem Mieder der Magd Susanna eine Brücke zu bauen, „um sie wieder aufzurichten“9. Es hilft nichts, sie bleibt, wie Susanna sich ausdrückt, platt wie ein Pfannkuchen. Walter ergreift die Verzweiflung, als er das hört, und er bittet Onkel Toby, seinen Bruder, ihn in sein Sehlafgemach zu führen. In größtem Schmerz wirft er sich auf sein Bett - und dann folgt die erste Beschreibung der Pose, die er dabei einnahm. Die Formulierung ist in bewusstem und durchaus komischem Gegensatz zu Walters wildem Jammer von größter Pedanterie:

„Wie er aufs Bett fiel, empfing sein rechter Handteller seine Stirn, bedeckte größtenteils die Augen und sank mit dem Kopfe sacht hinab (sein Ellenbogen wich dabei zurücke), bis seine Nase die Steppdecke fühlte; - sein linker Arm hing fühllos über die Bettkante, seine Knöchel ruhten auf dem Henkel des Nachttopfs, der unter der Bettgardine hervorlugte, - sein rechts Bein (das linke hatt' er an den Leib gezogen) hing halb über dem Bettrand, dessen Kante ihm ans Schienbein drückte.“10

Sterne, s. Anm. 4, S. 262-292.

Eine von mehreren Stellen: ebd., S. 236 (31. Kap., 3. Buch): ,[...] so erkläre ich, daß ich mit diesem Wort eine Nase meine und nichts mehr oder weniger."

Ebd., S. 231 (27. Kap., 3. Buch).

10 Ebd., S. 233 (29. Kap, 3. Buch). 
Onkel Toby setzt sich dazu und sagt kein Wort. Damit schließt das 29. Kapitel des dritten Buches, im folgenden 30. Kapitel heißt es nur, dass die zerdrückte Nase allein nicht den unsäglichen Kummer von Walter erklären könne, und um nun das Übermaß seiner Trauer erhellen zu können, müsse er ihn erst einmal eine halbe Stunde auf dem Bett liegen lassen. ${ }^{11}$ Damit ist die Pose eingefroren.

Mit dieser Bemerkung sind alle folgenden Digressionen, die sich mit Nasenproblemen in jeder denkbaren Hinsicht beschäftigen, gerechtfertigt. Erklärungen für Walters übergroßen Kummer steuern sie natürlich nur indirekt bei. Slawkenbergius und seine überlieferte Geschichte werden vorläufig eingeführt, das geht bis zum 42. Kapitel so, mit dem das dritte Buch endet. ${ }^{12}$ Die 35-seitige Geschichte von Slawkenbergius folgt als Zwischenspiel. Sie wird in der Ankündigung mit einigem Recht eine Nasenkunde ${ }^{13}$ genannt. Direkt danach, ohne Überleitung, folgt das erste Kapitel des vierten Buches, es wird eingeführt mit dem Hinweis, dass das Wissen um die Nasen unaufhörlich im Gehirn von Walter herumspukt, und sogleich kommt Sterne auf die Bettpose zurück:

„[...] wie wäre es da möglich bei einem so erlesenen - war's eine echte Nase? $\mathrm{Da}$ ein Mann von so erlesenem Feingefühl wie mein Vater den Schock unten oder vielmehr droben, überhaupt und in irgendeiner anderen Stellung hätte aushalten können als in eben der, die ich beschrieben habe ${ }^{\text {“14 }}$,

lässt Sterne den Erzähler Tristram fragen und fährt fort:

„Werft Euch nur frisch ein Dutzend Mal aufs Bett - doch vergeßt nicht, erst einen Spiegel auf einen Stuhl daneben zu stellen, bevor Ihr's tut - Aber war des Fremden Nase denn nun eine echte Nase - oder war's eine falsche?"15

Das ist einigermaßen verwirrend. Das untere und obere Stockwerk bezieht sich auf der ersten Ebene natürlich auf Walters Haus, auf Parterre und ersten Stock, aber, man kann es nicht überlesen, auch auf Walters bzw. Tristrams Körper. Die Pose gilt es dann genau zu studieren, als wäre es eine einzuübende Theaterpose, sie wird auf absurde Weise festgezurrt, als wäre sie eine künstliche bzw. künstlerische Pose. Und schließlich sind wir plötzlich noch einmal in der Slawkenbergius-Geschichte, denn dort wurde die Debatte darüber geführt, ob seine Nase echt oder künstlich sei.

Noch einmal wird ein kleines Stück dieser Geschichte eingeschoben, dann folgt das zweite Kapitel des vierten Buches, und nun wird die Pose von Walter weiter beschrieben, nachdem er anderthalb Stunden, nicht wie angekündigt nur eine halbe Stunde - denn die Slawkenbergius-Geschichte war viel länger als erwartet - regungslos dort gelegen hatte,

\footnotetext{
Ebd., S. 234 (30. Kap, 3. Buch).

2 Ebd., ab dem 38. Kap, 3. Buch, S. 247 ff.

Ebd., S. 249 (38. Kap, 3. Buch).

Ebd., S. 293 (1. Kap, 4. Buch).

Ebd.
} 
,als ob ihn die Hand des Todes niedergeworfen hätte, erst dann begann er mit dem großen Zehe des Fußes, der über die Bettkante hing, auf der Zimmerdiele zu spielen; meines Onkel Toby's Herz ward ein Pfund leichter drob. - Nach wenigen Augenblicken kehrte auch das Gefühl in seine linke Hand zurück, deren Knöchel die ganze Zeit über auf dem Henkel des Nachttopfs geruht hatten - er stieß ihn etwas weiter hinter die Bettgardine - hob nach getaner Arbeit die Hand zum Busen und steckte sie hinein - machte Hem! " $16^{16}$

Onkel Toby ist über dieses Lebenszeichen höchlichst erleichtert, doch er wartet erst einmal ab, und so heißt es zu ihm: ,[...] so beschied er sich damit, das Kinn sanft auf das Querholz seiner Krücke zu stützen. ${ }^{\text {(117 }}$

Im sechsten Kapitel des vierten Buches erleidet Walter nach einem kurzen Gespräch mit Toby in der Tat einen Rückfall - was ganz wörtlich zu nehmen ist, denn er fällt noch einmal in seine zuvor schon zweimal beschriebene Pose zurück, wieder ist die Nase flach im Kissen, alle Gliedmaßen nehmen ihre zuvor innegehabte Position ein, doch Walter möchte sich endlich aus dieser todesähnlichen Lethargie befreien, was Sterne zu folgendem Einschub veranlasst, der seine eigenen Beschreibungen aufs Schönste zu konterkarieren scheint: ,-- Stellungen an sich machen nichts her, Madam, - im Übergang von einer Stellung zur ander'n - gleich wie die Vorbereitung und Auflösung einer Dissonanz in eine Harmonie“"18, um dann fortzufahren: „Aus dem nämlichen Grund führte mein Vater den Tanz mit der großen Zehe auf der Zimmerdiele erneut auf - stieß den Nachttopf noch ein bißchen weiter hinter die Bettgardine - machte Hem! [...] $]^{\text {‘19 }}$

Danach steht er auf und hält im folgenden Kapitel Onkel Toby eine Rede, bei der er die Pose des Sokrates aus Raphaels Schule von Athen einnimmt, wie ausdrücklich vermerkt wird. Später, im 17. Kapitel, wird der Leser noch einmal nachdrücklich an die Bettszene erinnert. ${ }^{20}$ Hat man all dies gelesen, so fragt man sich geradezu notwendig, warum im Himmel diese Ausführlichkeit, diese Stillstellung durch Einfrieren der Szene, die geradezu penetrante Beharrlichkeit mit der Wiederholung einzelner Motive wie der Nase im Kissen, dem dicken Zeh, der auf den Boden trommelt oder dem Nachttopf hinter dem Bettvorhang; auch dass Toby sich mit dem Kinn auf seinen Stock stützt, wird zweimal erzählt. Was soll die Betonung dieser im Prinzip irrelevanten Motive, warum scheint Sterne Wert darauf zu legen, dass wir sie uns einprägen? Warum macht er den Leser, der wissen will, was das soll, geradezu nervös, wo er doch gleichzeitig das Erkenntnis nicht Befördernde von Stellungen als solchen betont? Was soll uns diese Diskrepanz, diese Paradoxie demonstrieren?

Ich denke zweierlei. Zum einen soll uns die Beschreibung dieser bildhaften Figuration in allem Detail auf den Zitatcharakter des Geschilderten verweisen,

\footnotetext{
${ }^{16}$ Ebd., S. 295 (2. Kap, 4. Buch).

Ebd.

Ebd., S. 298 (6. Kap., 4. Buch).

Ebd.

20 Ebd., S. 314 (17. Kap., 4. Buch).
} 
allerdings kann es sich aufgrund der wiederholten und variierten $\mathrm{Be}$ schreibung auch durch das schrittweise Hinzufügen weiterer Details kaum um einen Text als Vorbild handeln, sondern nur um ein Bild, und zwar, wie immer bei Sterne, um ein Bild aus einem ganz anderen $\mathrm{Zu}-$ sammenhang, das jedoch mit seinem ganz anders gearteten Bedeutungsfeld dennoch auf das Bedeutungsfeld, auf das es zur Anwendung gekommen ist, abfärbt. Die Verwandtschaft der Figuration zwingt uns geradezu dazu, eine Sinnübertragung zu versuchen.

Zugleich aber - und dies markiert das sprachwissenschaftliche Problem, auf das Sterne uns hinweisen will - wird uns die Ambivalenz der Zeichen bewusst, wir können uns nicht darauf verlassen, dass sie ein eindeutiges Verständnis der Dinge und ihres Zusammenhanges stiften.

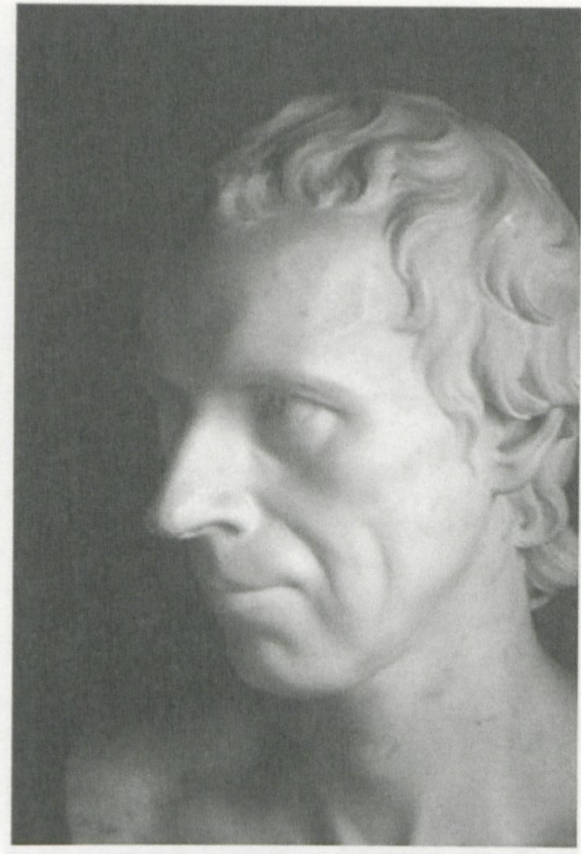

Abb. 1: Joseph Nollekens, Bildnis Laurence Sterne (ca. 1766), Marmorbüste, National Portrait Gallery, London Doch wie wäre ein etwaiges Vorbild auszumachen, wenn man nicht - das ist oft in der Sterne-Forschung bei literarischen Zitaten der Fall gewesen - auf einen augenöffnenden Zufallsfund stößt? Ich fürchte, es geht nur auf eine Sterne'sche Weise, durch Assoziation. Doch Assoziationen, nichts versucht Sterne nachdrücklicher zu demonstrieren, folgen keiner Systematik, vielmehr einer sehr eigenen, im Subjekt mit seinen Erfahrungen zu verortenden Logik, falls man überhaupt von Logik sprechen kann. John Locke hat geradezu panische Angst vor willkürlichen, alogischen Assoziationen. Doch Sterne ist gerade an den Antriebskräften für diese Art von Assoziationen interessiert und findet sie im Triebleben, der Sexualität der Menschen, später in abgeschwächter Form und damit weniger direkt im Gefühlsleben - das war zeitgenössisch eher akzeptierbar. So müssen wir, um den Assoziationsraum andeuten zu können, uns noch einmal den Kontext, in dem die Bettszene, gefärbt durch die Abschweifungen, ihr Vorkommen hat, klar machen und die Stichworte aufrufen, die damit markiert sind und zu ihnen versuchsweise assoziieren.

Die Bettszene steht im absoluten Zentrum von Tristram Shandy, im Kontext von Tristrams Geburt, über die der Text insgesamt kaum hinauskommt. Das Zerdrücken der Nase durch Dr. Slops Geburtszange ist vor allem im Kontext der Geschichte von Slawkenbergius und dessen Riesennase (Abb. 1) 


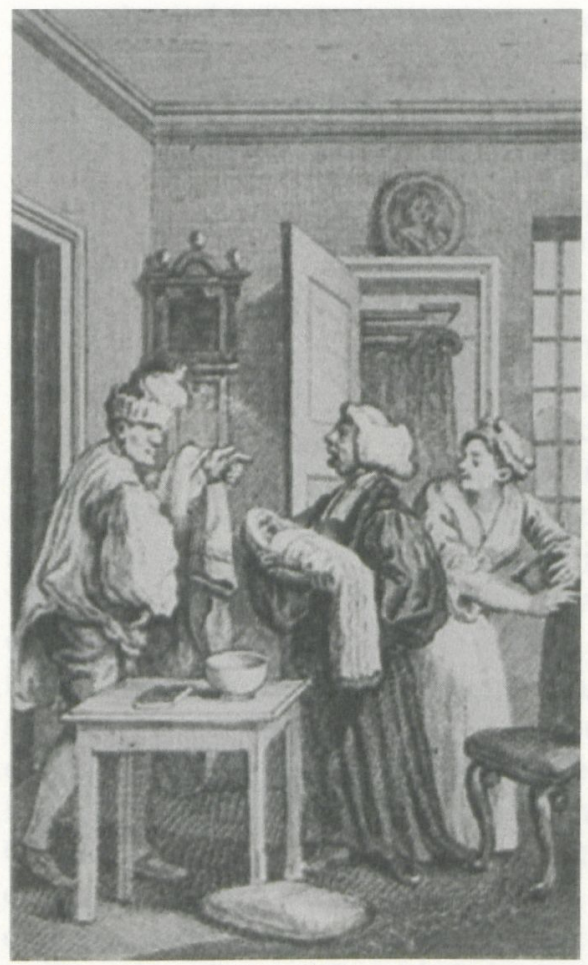

Abb. 2: François Ravenet nach William Hogarth, Frontispiz zum dritten Band von Tristram Shandy (1761), Kupferstich als (vermeintlicher) Ausweis höchster Potenz - erinnert sei an Sternes eigene Riesennase - nicht anders als ein Hinweis auf Tristrams Impotenz zu lesen, die ihre traurige Gewissheit erfährt, als Susanna den Knaben Tristram aus dem Fenster pinkeln lässt und unter der Verrichtung das Schiebefenster heruntersaust. So ist er unten und oben verstümmelt, was Rückschlüsse auch auf Onkel Tobys Unterleibsverletzung zulässt. Doch auch Walter in seiner Panik vorm wöchentlichen Ehevollzug reflektiert ohn' Unterlass die altersbedingte Impotenz. Alle Hoffnung hat er auf seinen Sohn gesetzt, doch er wird die Shandy'sche Linie nicht fortsetzen können - aufgrund dieser Einsicht wirft sich Walter aufs Bett und ist wie tot.

An dieser Stelle lohnt es sich, einen Blick auf Hogarths zweite Illustration zu Sterne (Abb. 2), die sich auf Tristrams Nottaufe im 14. Kapitel des vierten Buches bezieht, zu werfen. Walter liegt er-

schöpft in seinem Schlafzimmer auf dem Bett, als ihm gemeldet wird, sein neugeborener Sohn müsse dringend notgetauft werden, er sei schwarz angelaufen und drohe zu sterben, er solle schleunigst einen Taufnamen nennen. „Tristmegistus“, der dreimal Größte, aus mystisch-kabbalistischer Tradition soll sein hochtrabender Name sein, doch die Magd Susanna, die Walter geweckt hat, kann diesen komplizierten Namen nicht behalten. Sie meldet dem zur Taufe im Ankleidezimmer bereitstehenden Kurat in ihrer Aufregung den folgenden Namen: „'s ist Trist - Dingsbums. “21 Das bringt den Kuraten dazu zu bemerken, nur der Name Tristram beginne mit „Tris“ und dies sei auch sein eigener Name, und flugs tauft er das Baby auf ebendiesen Namen. ${ }^{22}$ Kaum ist dies erfolgt, stolpert Walter halb angezogen in den Raum, er hat im Dunkel seines Schlafzimmers seine Hose nicht finden können, flüchtig hat er schließlich die Hose übergestreift, nur ein Knopfloch geschlossen und auch dies nur halb, so geht der Knopf wieder auf, und er droht die Hosen zu verlieren, den

${ }^{21}$ Ebd., S. 309 (Kap. 14, 4. Buch).

22 Ebd., S. 309 f. 
Kampf um die Hosen scheint er nicht gewinnen zu können Sterne kennt offenbar auch diese bildliche Tradition (Abbildung 3). ${ }^{23}$ Die Nachtmütze hat Walter noch auf dem Kopf, den Schlafrock über dem Arm. $\mathrm{Er}$ ist in jeder Hinsicht zu spät gekommen. Genau diesen Moment zeigt Hogarth.

Warum wollte Sterne gerade ihn illustriert sehen? Schließlich kommt der Kurat Tristram nur in dieser Szene, für diesen einen Moment vor, und dennoch steht er im Zentrum von Hogarths Illustration. Walter hatte - zur Verblüffung des Lesers, denn zuvor war im Text berichtet worden, er sei längst tot - Pfarrer Yorick erwartet, doch an seiner Stelle

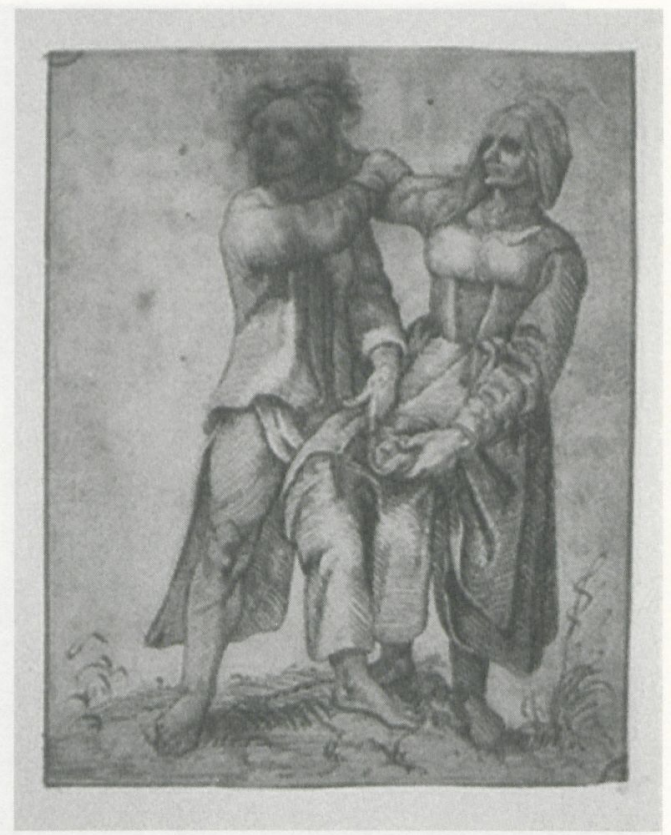

Abb. 3: Niederländische Zeichnung des 16. Jahrhunderts, Der Kampf um die Hosen taucht der Kurat, der bloße Stellvertreter, auf. ${ }^{24}$ Offenbar, nur Melvyn New hat beiläufig darauf hingewie$\operatorname{sen}^{25}$, war er Stellvertreter in verschiedener Hinsicht. Dass Tristram auf seinen Namen getauft wird, spricht für seine Vaterschaft. Offenbar hatte er ein Verhältnis mit Walters Ehefrau, wie er auch, so wird nahegelegt, ein Verhältnis mit Susanna hatte, die wiederum auch um Trim, den Burschen von Onkel Toby, keinen Bogen gemacht hat. Die Tatsache, dass Walter die Hosen verliert, spricht ebenso für die Berechtigung der Annahme von der Vaterschaft des Kuraten, wie auch auf Hogarths Illustration die hier überraschend wieder erscheinende Standuhr, die eigentlich ins Wohnzimmer gehört, wie die erste Illustration von Hogarth (Abb.4) gezeigt hat. Wieder erscheint sie über Walter, hier jedoch ist sie blind, ohne Zeiger, kann nicht auf den Ehevollzug verweisen. So ahnen wir auch Walters Problem. Offenbar ist sein permanentes gelehrtes Räsonieren bloße Ersatzbefriedigung, es hat ihn der Natur entfremdet, der allein das Personal in Walters Haus folgen kann. So ist die Rede von

${ }^{23}$ Sigrid Metken, Der Kampf um die Hose - Geschlechterstreit und die Macht im Haus, Frankfurt am Main, 1996.

24 Sterne, s. Anm. 4, S. 309 (Kap. 14, 4. Buch).

25 Melvyn New, Tristram Shandy. A Book for Free Spirits, New York, 1994, S. 47 f.; Sterne selbst weist versteckt darauf hin, indem er den Kuraten zwar nicht noch einmal auftauchen lässt, doch wird er in sehr bezeichnender Weise im Gespräch zwischen Walter und Onkel Toby genannt: Sterne, s. Anm. 4, S. 315 (18. Kap., 4. Buch). 


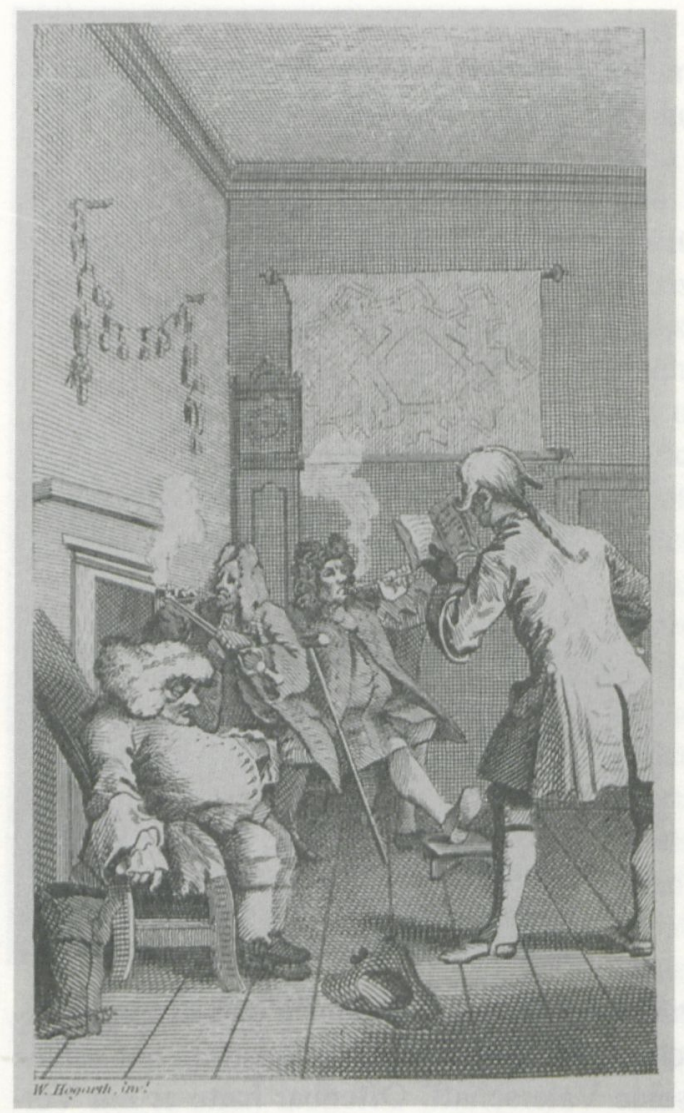

Abb. 4: François Ravenet nach William Hogarth, Frontispiz zum ersten Band von Tristram Shandy (1760), Kupferstich unerfülltem und erfülltem Trieb, von Betrug, Vorspiegelung falscher Tatsachen, von Potenz und Impotenz, von Verstümmelung und todähnlichem Schlaf.

Als ich mir dies klargemacht hatte und die von Sterne herausgestrichenen Details der Beschreibung der Bettszene noch einmal rekapituliert hatte, ahnte ich plötzlich zumindest, welches Thema die Folie abgegeben haben könnte: Samson und Delilah. ${ }^{26}$ Die berühmteste Fassung des Themas stammt zweifellos von Rubens (Abb. 5), gemalt 1609/10 für den Antwerpener Bürgermeister Rockox, weit verbreitet als Erfindung durch den seitenverkehrten Nachstich von Jacob Matham (Abb. 6). Die lateinische Beischrift hebt unter anderem auf die List der Weiber ab, die selbst die stärksten Männer besiegen und ins Verderben führen. ${ }^{27}$ Rubens zeigt den Moment, als Samson nach der

Liebesnacht mit Delilah in ihrem Schoß vorm Bett auf einem weichen Teppich eingeschlafen ist. Ein Jüngling schneidet ihm das Haupthaar, in dem, wie Delilah nach langem bohrenden Fragen schließlich von Samson erfahren hat, seine Kraft liegt. Mit größter Vorsicht geht er vor, um den kraftstrotzenden, unbesiegbaren Samson nicht zu wecken. Eine alte Frau hinter Delilah im klassischen Typ der Kupplerin leuchtet ihm bei der nächtlichen Szene mit einer Kerze. Die Tür zum Gemach ist bereits zum Teil geöffnet, Soldaten warten vorsichtig darauf, über Samson herfallen zu können. Über dem Kopf von Samson in einer Wandnische an der Rückwand findet sich die

${ }^{26}$ Zur Thementradition: Madlyn Kahr, ,Delilah“, in: The Art Bulletin 54 (1972), S. 282-299.

27 Roger-Adolf d'Hulst und Marc Vandenven, Rubens. The Old Testament, London, 1989, Kat.Nr. 31, S. 107-115, zum Nachstich von Matham: S. 108; Ausst.-Kat. Rubens. A Master in the Making, hrsg. v. David Jaffé, National Gallery, London, 2005, Kat.-Nr. 77, S. 166-168, zur thematischen Vorbereitung und zum Nachstich, ebd., Kat.-Nr. 72-76, S. 159-163. 


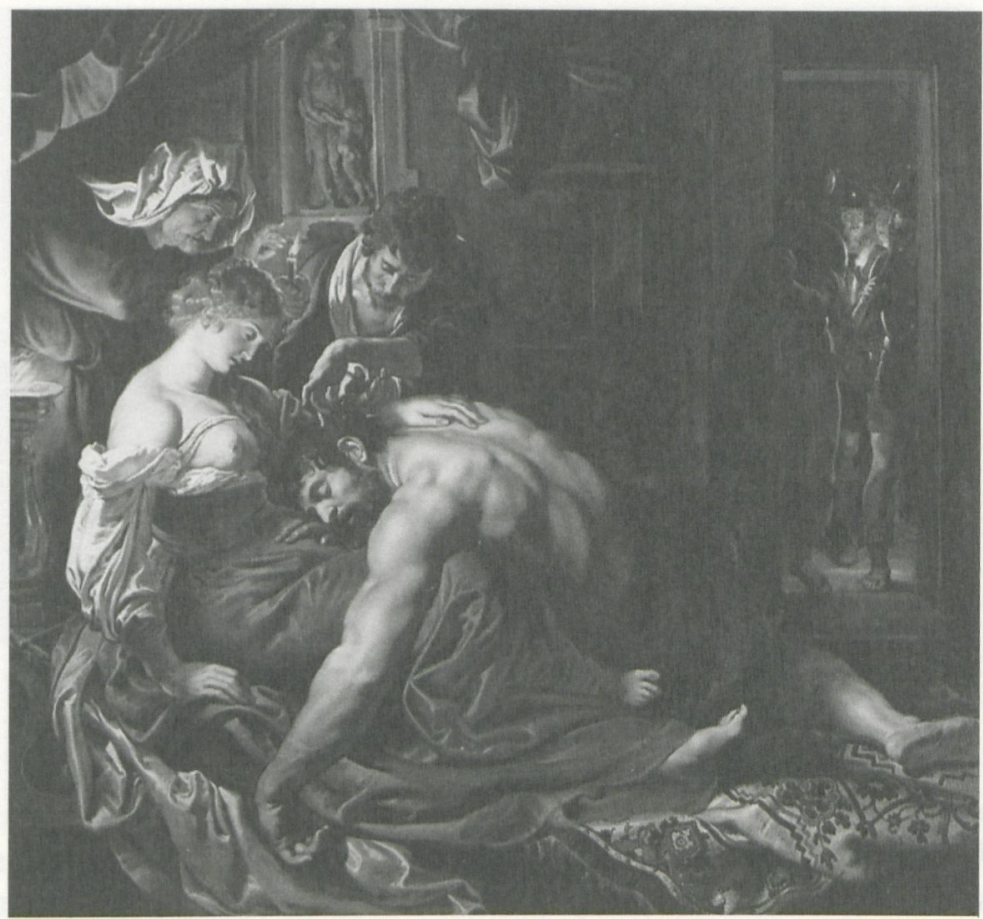

Abb. 5: Peter Paul Rubens, Samson und Delilah (um 1609), Öl auf Leinwand, National Gallery, London

Figurengruppe von Venus und Amor. Vergleicht man Gemälde und Stich, so stellt man fest, dass der Stecher hier die auffälligste Veränderung vorgenommen hat. Im Gemälde reckt Amor sich zu Venus hoch, sucht ihren Trost, ihr Gewand bedeckt ihren Unterleib und ihre Beine. Sie hält mit der Rechten Amors linke Hand, hat ihm ihre Linke beruhigend auf den Kopf gelegt. Bei Matham dagegen scheint Amor nicht um Trost nachzusuchen, sondern eher Venus' Gewand vor der Scham entfernen zu wollen, ohnedies verhüllt es sie nur dort. So ist der Nachstich expliziter. Kein schlechtes Gewissen über das Angerichtete scheint Amor erfasst zu haben, vielmehr scheint er noch die erotische Dimension der Szene verstärken zu wollen.

Nun weist die Rubens'sche Fassung des Themas vor allem in Gestalt des Samson nicht sehr viel Übereinstimmung mit Sternes Beschreibung der Pose Walters auf. Doch eine Erinnerung an die großen Van-Dyck-Ausstellungen in Washington 1990 und in London 1999 ließ vorm geistigen Auge van Dycks jugendliche Variante auf Rubens' Bild (Abb. 7) auftauchen. ${ }^{28}$ Und hier sind

28 Ausst.-Kat. Anthony van Dyck, hrsg. v. Arthur K. Wheelock, Jr., Susan J. Barnes und Julius S. Held, National Gallery of Art, Washington, 1990, Kat.-Nr. 11, S. 103-105; Ausst.-Kat. Van Dyck 1599-1640, hrsg. v. Christopher Brown und Hans Vlieghe, Koninklijk Museum 


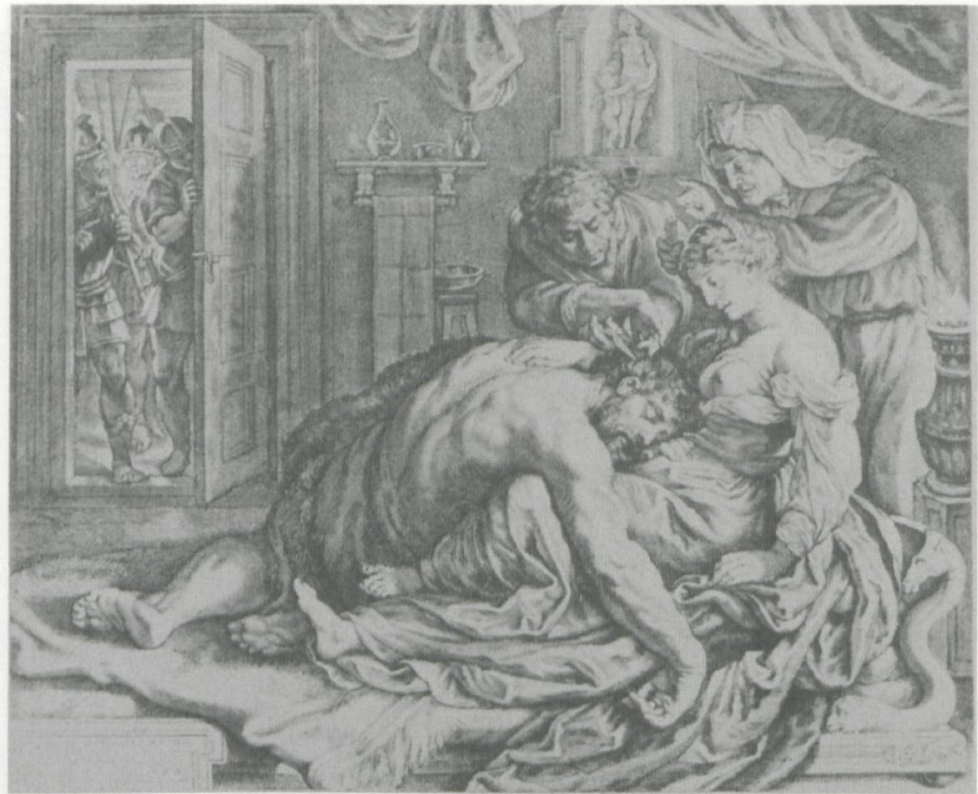

Abb. 6: Jacob Matham nach Peter Paul Rubens, Samson und Delilah, Kupferstich

die Übereinstimmungen frappant. Van Dyck hat das Repertoire der Szene weitgehend von Rubens übernommen, sucht jedoch für jedes Motiv seine eigene leichte Variante. Im Sinne des Rubens'schen Nachstiches ist die Szene seitenverkehrt zum Rubens'schen Gemälde angeordnet. So wird der Rubens'sche Stich der Ausgangspunkt van Dycks gewesen sein.

Statt im Dunkel eines nächtlichen Raumes mit flackerndem, das Drama verstärkendem Licht wie bei Rubens hat van Dyck die Szene halb im Freien angesiedelt. Delilah lehnt mit dem Rücken am schweren Überwurf ihres Sündenlagers. Samson und Delilah erscheinen wie vom Lager heruntergerutscht, der Bettüberwurf ist ihnen gefolgt, fließt im Bildvordergrund wie der Teppich bei Rubens am flachen Sockel des Gemaches herunter, bei van Dyck deckt er auch noch die linke Schulter von Samson. Dieser hat, und hier kann man Sternes Beschreibung für Walter benutzen, die Pose gänzlicher Erschöpfung eingenommen: „Wie er aufs Bett fiel, empfing sein rechter Handteller seine Stirn, bedeckte größtenteils die Augen und sank mit dem Kopfe sacht hinab (sein Ellenbogen wich dabei zurücke), bis seine Nase die Steppdecke fühlte ". ${ }^{29}$ Walters großer Zeh, der über die Bettkante hängt, weist bei van Dyck entschieden betont über die Kante des flachen Sockels. Während er bei Walter

voor schone Kunsten, Antwerpen; Royal Academy of Arts, London, München, 1999, Kat.Nr. 21, S. 136-139.

29 Sterne, s. Anm. 4, S. 233 (29. Kap., 3. Buch). 


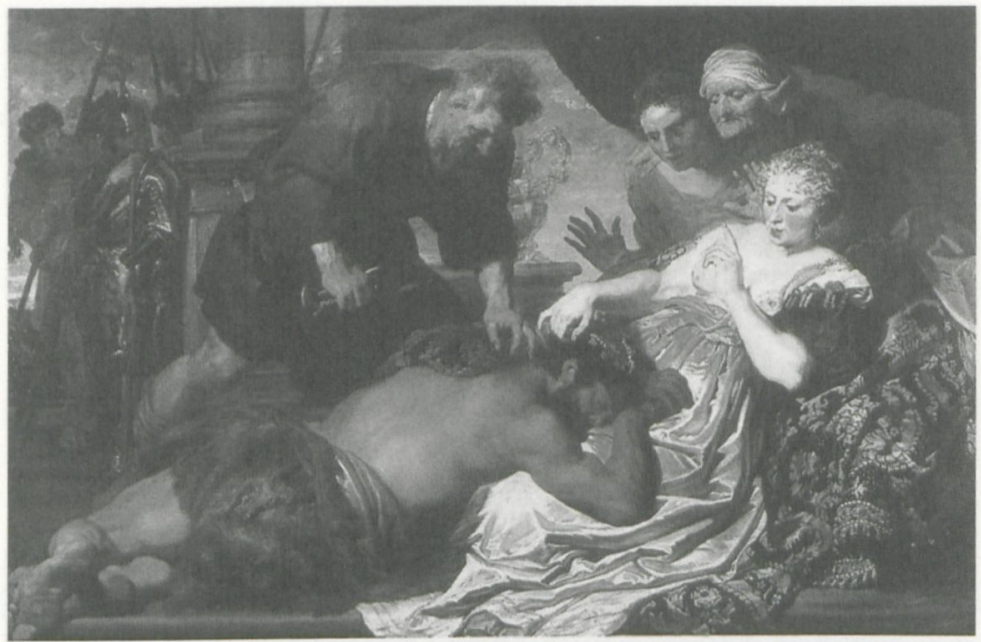

Abb. 7: Antonis van Dyck, Samson und Delilah (1619/20),

Öl auf Leinwand, Dulwich Picture Gallery, London

beginnt, auf den Boden zu klopfen, ragt er bei van Dyck über die ästhetische Grenze in den Betrachterraum hinaus und erfährt auf diese Weise seine Verlebendigung. Der vorderste Soldat, vorsichtig abwartend, ob die Schur bei Samson Erfolg hat, hat seine Hand auf eine kurze Lanze gestützt und darauf das Kinn aufgelegt - wie Toby auf seinen Krückstock, als er abwartet, wie es mit Walter weitergeht.

Zuletzt sei ein Motiv betont, das Sterne vielleicht am allermeisten zur Transformation angeregt hat: Wenn oberhalb des Rubens'schen Samson die Gruppe von Venus und Amor einen Kommentar zum Geschehen abgibt, dann

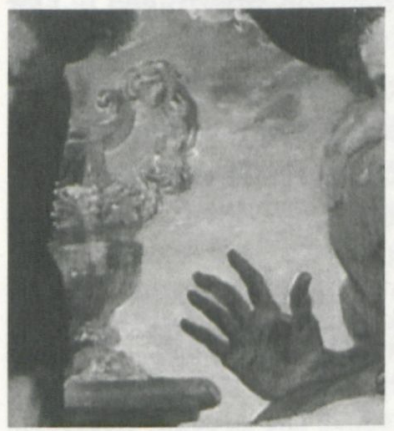

Abb. 8: Ausschnitt aus Abb. 7 hat van Dyck sie an entsprechender Stelle durch einen goldenen Weinkrater ersetzt, der wie der Nachttopf von Walter hinter den Bettvorhang gerückt ist. Entscheidend an van Dycks Krater ist der Henkel: Er wird von einem sich wild aufbäumenden Satyr mit erigiertem Glied gebildet (Abb. 8). Dieser Satyrhenkel befindet sich wieder exakt über dem Kopf des Samson und gibt einen beredten Kommentar zum Triebverlangen des biblischen Helden. ${ }^{30}$ Erinnert man sich daran, dass Walter die ganzen anderthalb Stunden, die er auf dem Bett lag, den Henkel des Nachttopfes mit den Knöcheln der Hand berührt, und imaginiert

${ }^{30}$ So auch: J. Douglas Stewart, Bespr. von Christopher Brown, Van Dyck, Ithaca, 1982, in: Revue d'art Canadienne/Canadian Art Review (RACAR) 13 (1986), S. 139-141, zur Satyrvase, einem Weinkrug, S. 140. 


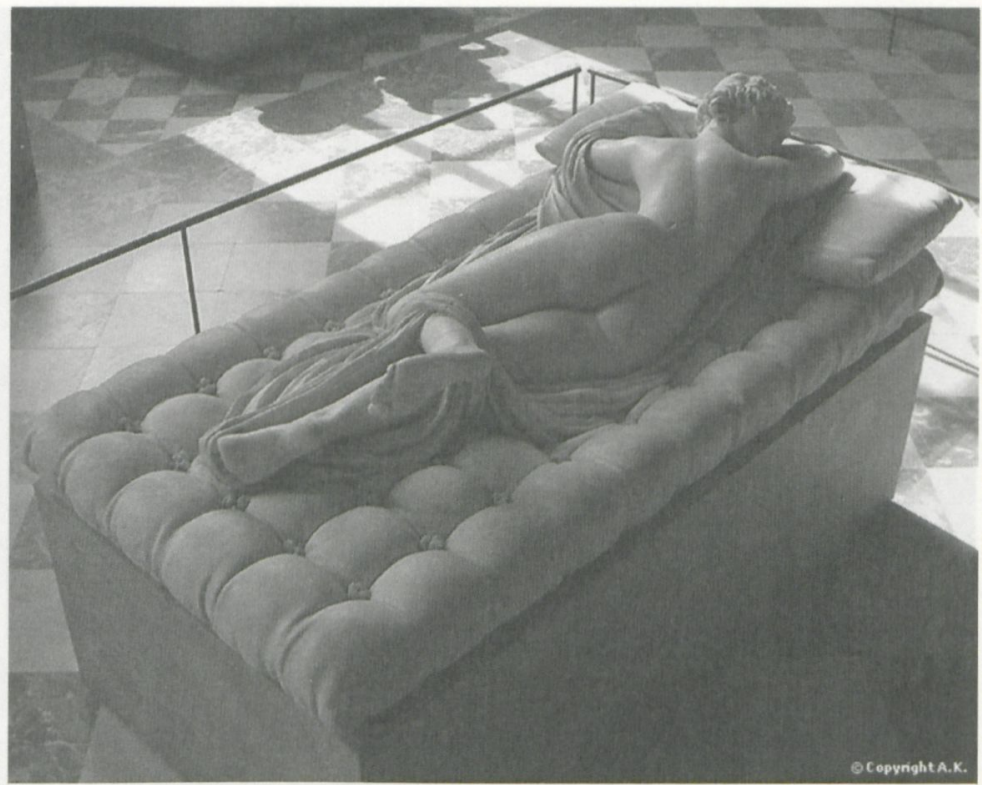

Abb. 9: Hermaphrodit, Rückansicht, Marmor, Louvre, Paris

sich den van Dyck'schen Henkel dazu, so ist das Resultat einigermaßen obszön.

Sollte Sterne gar die Herkunft der Pose des Samson bei Rubens und van Dyck realisiert haben, so verstärkt sich dieser Aspekt noch. Rubens hat in Italien den antiken Hermaphroditen (Abb. 9) gezeichnet, zu dem Bernini bekanntlich die Matratze geschaffen hat. Es ist von einiger Raffinesse und auch nicht ohne Ironie, dass Rubens und van Dyck die Pose des zarten Hermaphroditen auf den Leib des muskelbepackten Athleten Samson übertragen. ${ }^{31}$ Der Hermaphrodit wird traditionellerweise in grafischen Reproduktionen von hinten oder seltener von oben wiedergegeben, bei Perrier 1638 etwa in der einen, bei Maffei 1704 in der anderen Weise, wie ihn auch Rubens zeichnet. ${ }^{32}$ So sieht man seine schönen Rundungen und nur schwach seinen Brustansatz. Die Vorderansicht (Abb. 10), die die gesamte linke weibliche Brust, vor allem

31 Beobachtet von John Rupert Martin, „The Young van Dyck and Rubens“, in: Revue d'art Canadienne/Canadian Art Review (RACAR) 10 (1983), S. 37-43, zum Hermaphroditen als Vorbild für die Pose S. 38; zuvor bereits beobachtet von Horst Vey, Die Zeichnungen Anton van Dycks, Brüssel, 1962, Bd. 1, Text, S. 75; zum Hermaphroditen und zu seiner Rezeption: Francis Haskell und Nicolas Penny, Taste and the Antique. The Lure of Classical Sculpture 1500-1900, New Haven/London, 1981, Kat.-Nr. 48, S. 234-236.

32 Ausst.-Kat. Bernini Scultore. La nascità del Barocco in Casa Borghese, hrsg. v. Anna Coliva und Sebastian Schütze, Rom, 1998, Kat.-Nr. 9: Bernini, Ermafrodito, Abb. S. 125 schräg von vorn, Kat.-Nr. 10: Giovanni Francesco Susini, Ermafrodito, S. 126-133, Text der Katalognummern von Matthias Winner, dort Fig. 1 die Rubens zugeschriebene Zeichnung, Fig. 2 Perriers Stich von hinten, Fig. 4 Maffeis Stich von oben. 


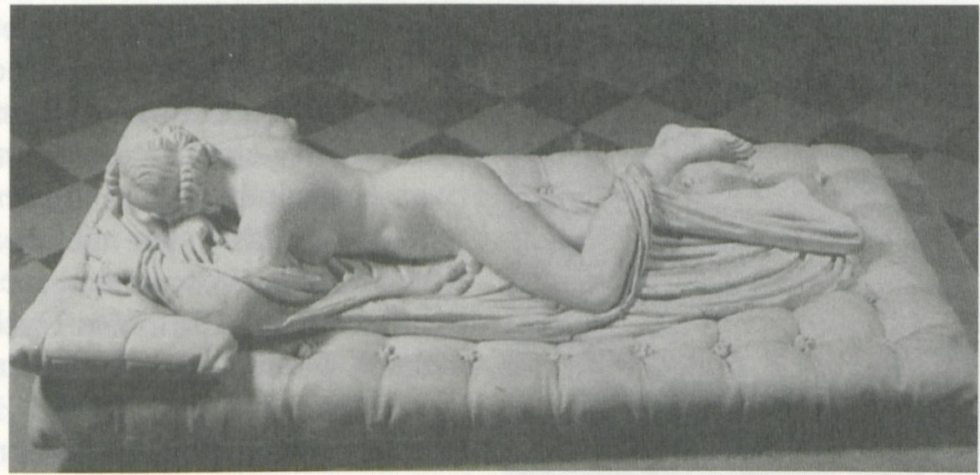

Abb. 10: Hermaphrodit, Vorderansicht, Marmor, Louvre, Paris

aber das erigierte Knabenglied zeigt, wurde offenbar als obszön in den Reproduktionswerken bewusst vermieden. Kaum eine Antike ist im Kleinformat in Marmor oder Bronze, selbst in Elfenbein häufiger kopiert und reproduziert worden als der Hermaphrodit. Seltener erscheint sie im Großformat. Gelegentlich, wie bei der kleinen Marmorkopie in Petworth in Sussex, ist der Penis bewusst weggelassen worden, so konnte man eine Nymphe in der Figur sehen. ${ }^{33}$ Wundervoll der für Lady Townshend überlieferte Ausspruch zu einer Kopie in der Mitte des 18. Jahrhunderts: Die Figur verkörpere ,the only happy couple she ever saw ${ }^{634}$. Die Reiseführer warnten vor ihrem die Schamgrenzen überschreitenden Anblick. Sterne dürfte besonders die kastrierte Version in Petworth animiert haben, schließlich widerfuhr Tristram ebendieses Schicksal. Letztlich mag sich gar die Idee zum trommelnden großen Zeh bei Sterne, aber auch schon zu van Dycks Trompe-l'EEil vom Hermaphroditen herleiten, denn bei ihm findet sich das höchst seltsame Motiv, dass die Zehen des linken FuBes in das Schlaftuch treten und es so anspannen (Abb. 11). In England wusste man auch um Berninis Matratze, die den Hermaphroditen erst eigentlich ins Bett befördert. Smollett, dessen Reisebericht aus Italien Sterne parodiert und den er dort in persona traf, hält in ebendiesem Bericht die Matratze für ,the most curious circumstance ${ }^{\text {c35 }}$ des ganzen Werkes.

Nimmt man den Zusammenhang von Sternes Bettszene und van Dycks Samson und Delilah als gegeben an - und kaum etwas dürfte dagegen sprechen -, so stellen sich zwei Fragen. Zum einen: Woher konnte Sterne das Bild kennen? Grafische Reproduktionen gab es nicht. Und zum anderen: Warum erschien Sterne gerade die Geschichte von Samson und Delilah für die Über-

33 Haskell und Penny, s. Anm. 31, S. 235.

34 Ebd. Eine derartige Bemerkung wird von Walpole überliefert: Horace Walpole's Correspondence with Sir Horace Mann, Bd. 2, hrsg. v. W. S. Lewis (The Yale Edition of Horace Walpole's Correspondence, Bd. 18), New Haven, 1954, S. 342 (Brief an Sir Horace Mann, 17. November 1743) bezieht sich auf die Bronzefassung im Besitz von Horace Walpole. 


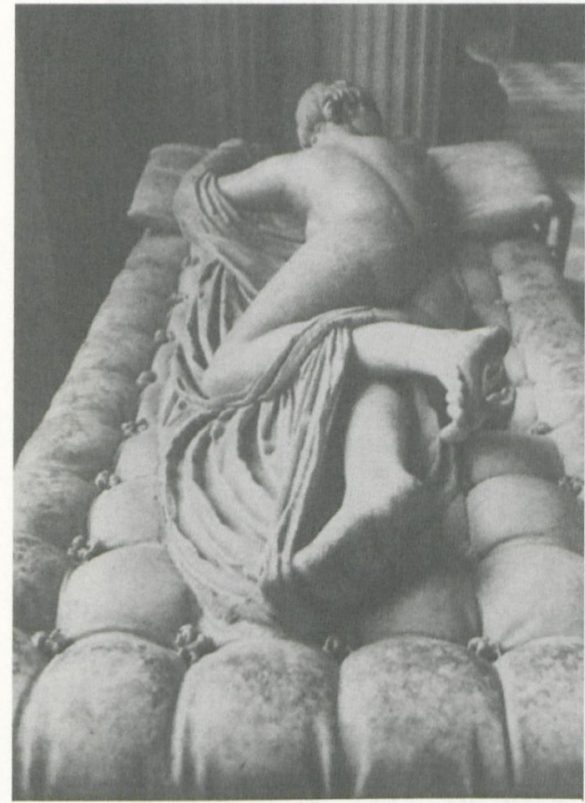

Abb. 11: Hermaphrodit, von den Füßen aus gesehen, Marmor, Louvre, Paris

tragung als besonders geeignet? Die theologische Bedeutung und die Exegesetradition waren ihm mit Sicherheit vertraut, nicht umsonst war er hauptberuflich Pfarrer. Die erste Frage lässt sich mit ein wenig Recherche überraschend einfach beantworten. Die Van-Dyck-Forschung weiß zur Provenienz zu berichten, dass das Bild 1711 im Besitz von David Armory in Amsterdam war und dort im sogenannten Armory Sale 1722 versteigert wurde. Der nächste quellenmäßige Beleg stammt von 1767 und führt das Bild als im Besitz von Sir Gregory Page an. ${ }^{36}$

Auf seinem Landsitz vor den Toren Londons konzentrierte Page seine hochbedeutende Gemäldesammlung, zu der Werke unter anderem von Claude, Poussin, Veronese, Rubens und eben van Dyck gehörten. Eine Liste seiner Bilder wurde 1761 in London and its Environs Described im ersten Band abgedruckt. ${ }^{37}$ Dieser sechsbändige London-Guide war schon vor dem offiziellen Publikationsdatum zugänglich. Er erschien bei den wichtigsten Londoner Verlegern Robert und James D. Dodsley - ebenden Verlegern der zweiten Auflage der ersten beiden Bände von Tristram Shandy aus dem Jahr 1760, die bei Sternes Anfrage zum Druck der Erstauflage noch zurückhaltend gewesen waren. Am Ende des Jahres 1760 publizierten die Brüder auch den dritten und vierten Band von Tristram Shandy, in dem sich die Beschreibung von Walters Position auf dem Bett befindet. So spricht vieles dafür, dass Sternes Verleger den Kontakt zu Sir Gregory Page herstellten und Sir Gregory zu den vierzehn hochbedeutenden Adligen und Honoratioren gehörte, von denen Sterne 1760 voller Stolz berichtet, dass sie alle sich darum gerissen hätten, ihn zu sich zum Dinner einzuladen und den Abend mit ihm zu verbringen. ${ }^{38}$ Bei Sir Gregory

36 Siehe oben Anm. 28.

37 London and its Environs Described, London, 1761, Bd. 1; siehe auch ,John Brushe, Wricklemarsh and the Collections of Sir Gregory Page“, in: Apollo 122 (1985), S. 364-371; vorher schon: Denys Sutton, „Aspects of British collecting, Part II“", in: Apollo 116 (1982), S. $358-420$.

38 Ross, s. Anm. 1, S. 246 f.; Laurence Sterne, The Letters, Teil 1: 1739-1764, hrsg. v. Melvyn New and Peter de Voogd (The Florida Edition of the Works of Laurence Sterne, Bd. 7), Gainesville (Fla.) u. a., 2009, Brief Nr. 49, 50, 52, 55. 
muss er sich Mitte 1760 Aufzeichnungen vor dem Van-Dyck-Gemälde gemacht haben, er muss erkannt haben, wie ideal seine Adaption geeignet war, seinem Text zu Tristram Shandy, der von Potenz und vor allem Impotenz und Kastration handelt, eine ganz besondere Färbung zu geben.

Wir hatten angemerkt, dass van Dyck - seinem jugendlichen Drange mag es geschuldet sein - das erotische Moment vor allem durch den phallischen Krater gegenüber Rubens noch verstärkt hat, zudem scheint die verschattete Hand der jugendlichen Dienerin mit dem Zeigefinger direkt auf das Glied des Satyrn hinzuweisen. Selbst wenn man schon im 18. Jahrhundert wissen konnte, dass Satyr und Satire etymologisch nicht denselben Ursprung haben, so bot sich doch das Spiel mit der Wortähnlichkeit an, zumal Sterne das englische „satire“ grundsätzlich als „satyr" schrieb. Für ihn ist die Aufladung der Satire mit dem Triebpotenzial des Satyrn eine ideale Möglichkeit, auf die verdrängten Anlässe menschlichen Handelns anzuspielen. Die Satire bei Sterne lebt vom Obszönen und Grotesken. ${ }^{39}$

Nun fragt sich, wie Sterne, um es so zu sagen, den Honig aus der Samsonund-Delilah-Geschichte saugt. Auf welchen Feldern kann die Kenntnis des Vorbildes Assoziationen auslösen? Als Pfarrer weiß Sterne sowohl um die typologische Bedeutung der Samson-und-Delilah-Geschichte wie um die exegetische Tradition, die entschieden auf die Auffassung der Bildtradition abgefärbt hat. ${ }^{40}$ Die Samson-Geschichte ist paradox angelegt. Einerseits ist Samson ein unkontrollierter Berserker und Wüterich, andererseits wird sein Leben typologisch auf Christus bezogen. Schon diese Grundkonstellation, die entsprechend auch für Delilah gilt, dürfte die Geschichte für Sterne faszinierend gemacht haben. Es ist wirklich extrem: Einerseits ist Samsons Liebe zu Delilah triebhaft, und er rennt in sein Verderben, andererseits wird seine Liebe in typologische Parallele gesetzt zu Christi Liebe zur Kirche. Einerseits kostet Samson die Liebesnacht mit Delilah bis zur Erschöpfung aus, wobei Delilah ihm nur das Geheimnis seiner Kraft entlocken will, andererseits präfiguriert die von Rubens und van Dyck dargestellte Szene, die Samson seine Kraft raubt und zu seiner nachfolgenden Blendung führt, die Grablegung Christi.

Allerdings: Schon in den Jüdischen Altertümern des Flavius Josephus wird einseitig Samsons sündiges Verlangen betont, und Delilah, die in der Typologie als Zerstörerin des Unglaubens gefeiert werden konnte, wird zur Hure. ${ }^{41}$

39 Siehe dazu Antonio Ballesteros González, „Digression and Intertextual Parody in Nashe, Sterne and Joyce", in: Laurence Sterne in Modernism and Postmodernism, hrsg. v. David Pierce und Peter de Voogd, Amsterdam, 1969, S. 60. Zur Satire als angemessener Gattungsbezeichnung siehe Sterne selbst, der sich an Lord Bathursts Einschätzung von 1760 erinnert: Ross, s. Anm. 1, S. 10; Sterne, Letters, s. Anm. 38, Teil 2: 1765-1768, Brief Nr. 196 an Eliza Drapper [London, März 1767], S. 540. Ein Beispiel für Sternes Schreibweise: „I think there is more laughable humour, - with equal degree of Cervantik Satyr - if non more than in the last": Sterne, Letters, s. Anm. 38, Brief 63 an Jane Fenton, Coxwould, 3. August 1760, S. 165; Pfister, s. Anm. 1, S. 34 (zur Etymologie von Satire).

${ }^{41}$ Ebd., S. $284-286$. 


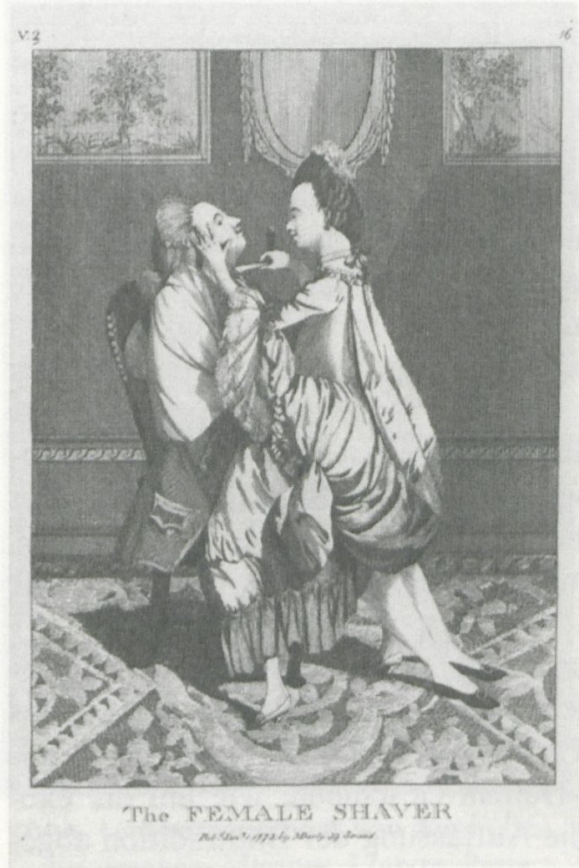

Abb. 12: Anonym, The Female Shaver (1773), Kupferstich

Biblisch lässt sich das rechtfertigen, genug Passagen lassen sich anführen, in denen die Frau allein als Verführerin gilt, um die der Mann besser einen Bogen machen sollte. ${ }^{42}$ In der Renaissance wird Delilah zu einer der vielen Verkörperungen männerzerstörender Weibermacht, und die Szene, in der Samson die in der Haarpracht verkörperte Kraft genommen wird, ist der direkte Ausdruck dieser Macht. ${ }^{43}$ In der Bibel heißt es, Delilah rief einen Mann, um den Akt des Haarescherens vorzunehmen, seit Flavius Josephus greift Delilah selbst zur Schere, und die Bildtradition folgt dem weitgehend. $\mathrm{Ob}$ ausgesprochen oder nicht, der Vorgang steht für Kastration. ${ }^{44}$ Josephus verbindet Samsons nicht $\mathrm{zu}$ bremsende Lust zudem mit $\mathrm{zu}$ großem Weingenuss, der auch Mahomet zum Verderben wurde. Lucas van Leyden hat beide Szenen dargestellt. ${ }^{45}$ Van Dyck verbindet in seinem Satyrkrater die Motive von Lust und Trunkenheit. Bei Sebastian Brant wird Samson zum Narren, die Moral wird eindeutig, und es ist kein Wunder, dass etwa bei Heemskerck Samson in der Pose des trunkenen Noah im Schoß von Delilah liegt. ${ }^{46}$ In der niederländischen Emblematik gar wird Delilahs Schoß sprichwörtlich für den Gang zu den Huren, und die symbolisch vollzogene Entmannung Samsons stellt nur eine gerechte Strafe dar. ${ }^{47}$

Der Bibeltext dagegen, und dies dürfte Sterne in seinen Grundüberzeugungen bestärkt haben, ist alles andere als explizit, er ist bedeutungsoffen, lässt diese und jene Lesweise zu und ist gerade damit umso wirkungsvoller. Samson ist zugleich Held und Sünder, Delilah folgt ihrer göttlichen Vorherbe-

\footnotetext{
Paulus, 1. Brief an die Korinther 7,1.

43 Kahr, s. Anm. 26, S. 286.

44 Ebd., S. 288, 299.

45 Ausst.-Kat. The Prints of Lucas van Leyden \& His Contemporaries, hrsg. v. Ellen S. Jacobowitz und Stephanie Loeb Stepanek, National Gallery of Art, Washington, 1983, Kat.-Nr. 11 und 12 (Mahomet), S. 60-63; Kat.-Nr. 34, S. 110-112; Kat.-Nr. 63, S. 176 f. (Samson und Delilah).

46 Kahr, s. Anm. 26, S. 287 (Brant), S. 290-292 (Heemskerck).

47 Ebd., S. 296.
} 
stimmung und ist zugleich Hure. In beiden spiegelt sich, protestantisch verstanden, die Unausweichlichkeit des Schicksals, das Wissen um unsere unaufhebbare Sündhaftigkeit bei gleichzeitiger durchaus paradoxer, allerdings bloßer Hoffnung auf Erlösung.

Dass das Haareschneiden mitnichten erst ab Freud als Zeichen von Kastrationsangst verstanden wird, vermag die Bildtradition des 18. Jahrhunderts zu lehren, besonders die englische Karikatur. Doch auch Sterne, ganz sicher in Kenntnis der englischen Bildtradition, macht das Motiv in Tristram Shandy zum Thema. Es sei hier zudem die durchaus gewagte, kaum durch direkte Evidenz abgesicherte These geäuBert, dass Goyas Obsession in Bezug auf dieses Thema sich nicht nur aus seiner nachweislichen Kenntnis der

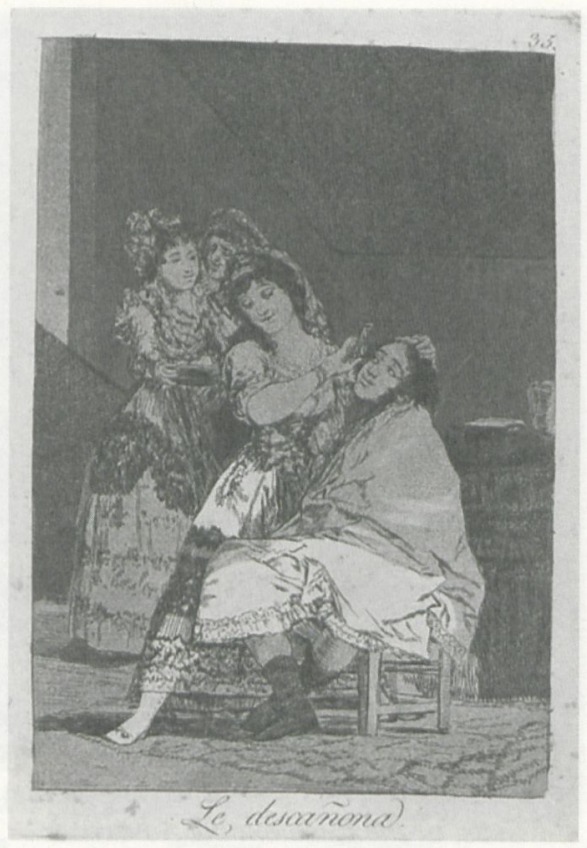

Abb. 13: Francisco de Goya, Capricho 35, La descañona (1799), Radierung und Aquatinta englischen Karikaturentradition herleiten lässt, sondern womöglich auch aus der Lektüre von Tristram Shandy, für die ihm allerdings nur die französische Übersetzung von 1777 bzw. 1784 zur Verfügung gestanden hätte. ${ }^{48}$

Nur zwei Beispiele aus der breiten englischen Tradition seien kurz angeführt, sie dürften Goya geläufig gewesen sein. Eine anonyme Karikatur von 1773 (Abb. 12) prägt einen lang anhaltenden Typus. ${ }^{49}$ Der, wie auch der Titel des Blattes lautet, ,female Shaver" sitzt mit weitem Kleid auf dem Schoß des zu Barbierenden, den erfreut dies, seine Hände dürften sich unter ihrem Rock befinden. Beide lächeln, doch sie hat die Rasiermessergewalt, und das Messer ist bereits an seine Kehle gesetzt. Goya folgt dieser Tradition in seinem Capricho 35 (Abb. 13) beinahe wörtlich, das Blatt trägt bei ihm den bezeichnenden Titel Le descañona, „Sie rupft ihn“, was durchaus doppeldeutig ist.

Wichtiger für uns ist eine Karikatur, die zwar um einiges nach Sternes Text erschienen ist, die Dinge aber auf den Punkt bringt. Sie ist betitelt: Cap.tn Puff, in the Suds, or the Fashionable Advertiser - Loosing his W[h]iskers

48 Laurence Sterne, La vie et les opinions de Tristram Shandy, übers. v. M. Frénais, Yorck/Amsterdam, 1777, 2. Ausgabe, London, 1784.

49 Reva Wolf, Goya and the Satirical Print in England and on the Continent, 1730 to 1850, Boston College Museum of Art, Chestnut Hill, 1991, Kat.-Nr. 33 und S. 51-57: Anonymous [Mary or Matthew Darly?], The Female Shaver, 1773. 


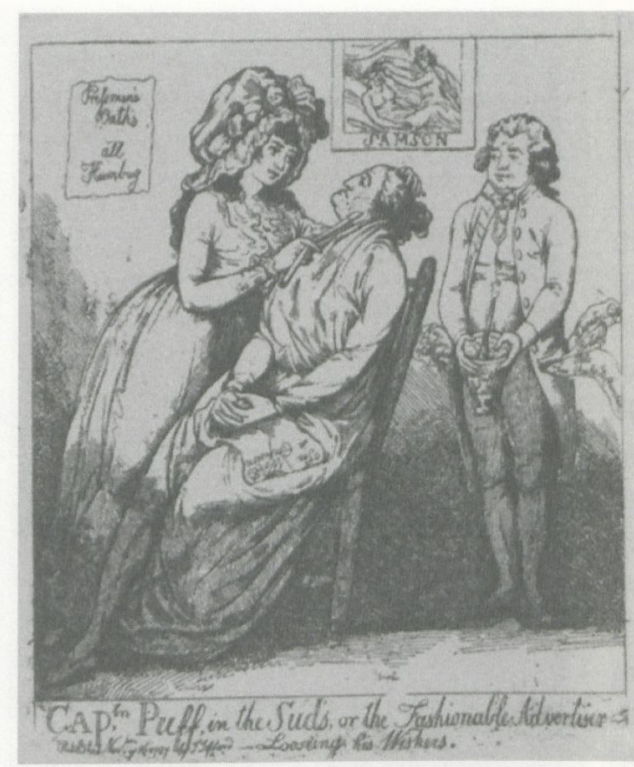

Abb. 14: Anonym, Cap.tn. Puff, in the Suds, or the Fashionable AdvertiserLoosing his Whiskers, Radierung

(Abb. 14) von 1787. ${ }^{50}$ Wieder führt bei diesem Pressevertreter, der nur aufdringliche Werbung verbreitet, eine hochattraktive Frau das Rasiermesser, es soll ihm seine „whiskers“, seinen Backenbart, entfernen, doch wieder ist das Messer gefährlich nahe an der Kehle, und sie hält mit der Linken seinen Kopf zurück, es ist kein Entkommen - auch Goya ließ sich bei genanntem Capricho dieses Motiv nicht entgehen. Über dem Kopf des verlogenen ,pressman“ findet sich an der Wand eine Darstellung von Samson und Delilah, just schert sie ihm darauf das Kopfhaar. So haben wir in der Karikatur einen betrogenen Betrüger, dem es schlecht ergehen wird.

Nun spielen die ,whiskers“" auch bei Sterne eine besondere Rolle. Am Beginn des fünften Buches, unmittelbar nachdem er im ersten Kapitel, Burton zitierend, über die Zitatpraxis reflektiert hat und den Plagiatoren die Krätze an den Hals wünscht, wird er eben dadurch an die Geschichte der (wie es in den deutschen Übersetzungen heißt) Zwickel- oder Knebelbärte erinnert, die er sofort unter dem Titel „Upon Whiskers" inseriert. ${ }^{51}$ Selbst wenn die Sterne-Forschung zu Recht bemerkt, dass es gänzlich unklar bleibt, was denn die „,whiskers“ jenseits der Zwickelbärte bedeuten könnten, so legt Sterne doch alles darauf an, dass wir danach fragen und spekulieren. ${ }^{52}$ Immerhin gibt es Anspielungen, dass auch mit den Zwickelbärten etwas Unanständiges gemeint ist, allen gegenteiligen Behauptungen zum Trotz: „[...] so gewiß als Nasen immer noch Nasen und Knebelbärte immer noch Knebelbärte sind (die Welt mag nur immer frisch das Gegenteil behaupten) [...]. ${ }^{\text {c53 }}$ Wenn es in der folgenden Bartgeschichte zu dem Ausruf kommt: „Aber er hat keinen Knebelbart [...]“ (,no whiskers“ im Eng-

50 Ebd., Kat.-Nr. 34 und S. 51-57: Anonymous, Cap.tn Puff, in the Suds, or the Fashionable Advertiser-Loosing his Whiskers, 1787.

51 Lawrence Sterne, Das Leben und die Ansichten Tristram Shandys, übers. v. Rudolf Kassner, überprüft und revidiert, München, 1964, (1. Kap., 5. Buch), S. $403-409$ („Zwickelbärte“); Sterne, s. Anm. 4, (1. Kap., 5. Buch), S. 368-372 („Knebelbärte“).

52 New, s. Anm. 25, S. 123.

53 Sterne, s. Anm. 4, S. 368 (1. Kap., 5. Buch). 
lischen $)^{54}$, dann wissen wir aus Kenntnis der Nasengeschichte eben doch, was gemeint sein soll. Sterne macht es uns insofern bewusst schwer, zu einem sicheren Urteil in der Zwickelbartfrage zu kommen, als er nur vom Bart und nicht vom Bartscheren spricht. Die bloße Benennung des Gegenstandes und nicht des mit dem Gegenstand womöglich verbundenen Vorgangs fordert uns auf, das fehlende Zwischenstück zur Bedeutungsgebung zu ergänzen, eine Verschiebung in semiotischer Hinsicht vorzunehmen, von der wir wissen, dass ihr etwas Willkürliches anhaftet. Und auf ebendieses Willkürliche legt Sterne Wert, weil er uns nicht nur den Schwarzen Peter zuschiebt, sondern uns uns selbst ertappen lässt, denn wir sind es, die das Unanständige hinter den „whiskers" erkennen möchten und vermuten. Unsere versteckte Lust kommt ans Licht unseres eigenen Bewusstseins und kann uns beschämen, aber auch zur

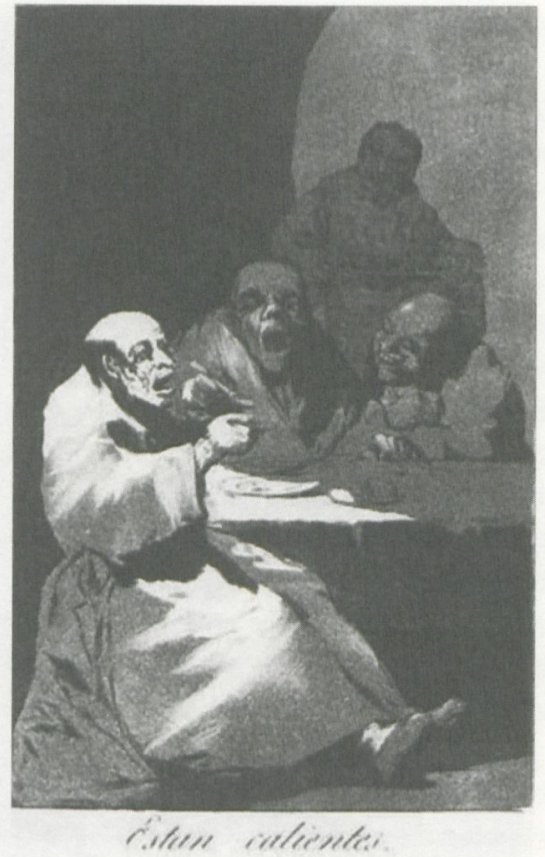

Abb. 15: Francisco de Goya, Capricho 13, Estan calientes (1799), Radierung und Aquatinta Reflexion über die Mechanismen von Erregung führen.

Wenn denn Goya Sterne gelesen hat, dann hat er dieses Satyrspiel durchschaut: Eine Fülle von Motiven in den Zeichnungen und Caprichos von Goya scheint bei Sterne vorgeprägt. Im Madrider Skizzenbuch findet sich eine Zeichnung, betitelt „Sie träumt von einem Schatz“, auf der eine auf dem Bett liegende, träumende Frau ihren Finger in den vorm Bett stehenden Nachttopf taucht, was offenbar den Traum auslöst ${ }^{55}$ - und an Walters eineinhalbstündiges Berühren des Nachttopfes erinnert. Capricho 13 lautet estan calientes, „Sie sind heiß" (Abb. 15), vermeintlich bezieht sich das auf das zu heiße Essen der um einen Tisch versammelten Mönche, doch trägt das „Sie sind heiß ${ }^{\text {c56 }}$ auch unverkennbar eine sexuelle Konnotation, und wieder kann sich der Betrachter bei seiner nicht zwingenden Assoziation - denn nichts auf dem Capricho gibt einen direkten Hinweis, allenfalls die Gier der Mönche ist angesprochen - er-

54 Ebd., S. 370; Laurence Sterne, The Life and Opinions of Tristram Shandy, Gentleman, hrsg. v. Ian Campbell Ross, Oxford, 2000, S. 277 (1. Kap., 5. Buch).

55 Pierre Gassier, The Drawings of Goya. The Complete Albums, London, 1973, Skizzenbuch B (Madrid), Nr. B.66, Abb. S. 98, Kat. S. 132: Sueña de un tresoro, 1796-1797.

56 Ausst.-Kat. Eleanor A. Sayre (Hrsg.), The Changing Image. Prints by Francisco Goya, Museum of Fine Arts, Boston, 1974, Kat.-Nr. 48 und 49, Capricho 13. 


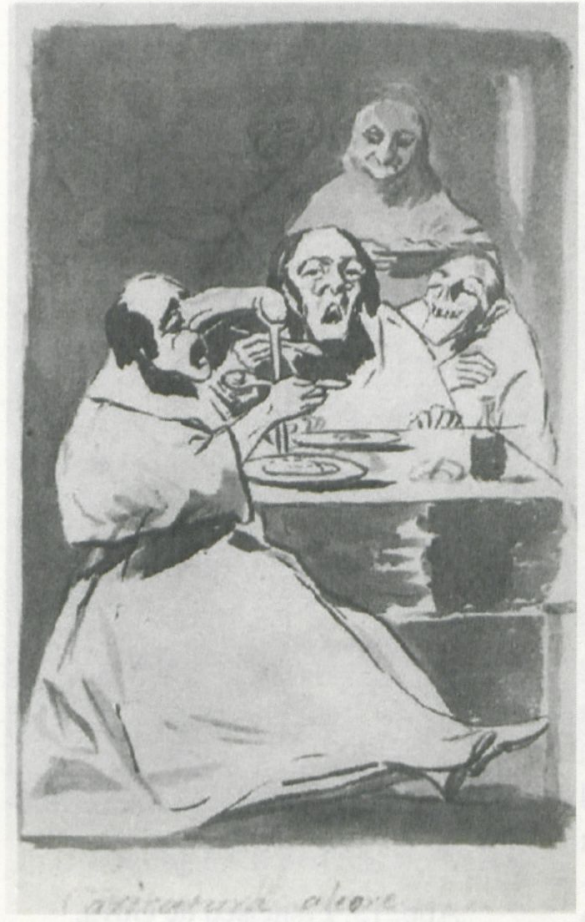

Abb. 16: Francisco de Goya, Caricatura alegre, Zeichnung, Madrider Album B,

S. 63, 1796/97, Prado, Madrid

tappt fühlen. Dass Goya jedoch nichts anderes im Sinn hatte, macht die Vorzeichnung aus dem Madrider Album B, Seite 63 (Abb. 16), entstanden 1796/97, mehr als deutlich. ${ }^{57}$ Der uns nächste gefräßige Mönch hat eine riesige Nase in Penisform, die so groß ist, dass sie von einem krückenartigen Ständer gestützt werden muss, damit sie nicht herabhängt und die Essbefriedigung verhindert. Bezeichnenderweise ist das Blatt betitelt Caricatura alegre.

Noch einmal in entsprechendem Zusammenhang verwendet Goya den Karikaturenbegriff, wieder im Album B, nun auf Seite 58, hier lautet der Titel: „Die Ehefrau fragt den Ehemann nach einer Erklärung ${ }^{458}$, und der Ehemann hat wieder eine gewaltige, nun gänzlich herabhängende Penisnase, zugleich greift er sich in die Hose. Der Grinsende im Vordergrund macht eine CornutoGeste - offenbar hat er dem impotenten Ehemann Hörner aufgesetzt. Im davon abgeleiteten publizierten Capricho 54 ist Goya, was diesen Zusammenhang angeht, etwas zurückhaltender, letztlich ist der Hinweis aber doch unverkennbar. Das Blatt heißt El Vergonzoso, „Der Schamhafte ${ }^{659}$, es folgt der in Spanien in den populären Bilderbögen weit verbreiteten Tradition der „Verkehrten Welt ${ }^{\star 660}$. Denn der Brei essende, zentral auf uns ausgerichtete Mann trägt seine Hosen als Kapuzenjacke. Durch den Schlitz schaut sein Gesicht, wieder verunziert durch eine Penisnase. Er schämt sich dafür, dass er im Gesicht trägt, was in die Hose gehört. Was diese Goya'schen Blätter, die noch zu vermehren wären, deutlich machen können, ist zum einen, dass die Nasenmetapher weit verbreitet ist, und zum anderen, dass Satire und Karikatur den Ort markieren, an dem sie zur Sprache kommen kann. Vielleicht sollte man auch

Gassier, s. Anm. 55, Nr. B.63, Abb. S. 95, Kat. S. 131: Caricatura alegre, 1796-1797.

58 Ebd., Nr. B.58, Abb. S. 90, Kat. S. 130: Caricat/Le pide cuentas la mujer al marido, 17961797 .

59 Alfonso E. Pérez-Sanchez und Julián Gállego, Goya: Das druckgraphische Werk, München/New York, 1995, Kat.-Nr. 54, S. 64; Barbara Kornmeier, Goya und die populäre Bilderwelt, Frankfurt am Main, 1999, S. 79-84.

60 Kornmeier, s. Anm. 59, S. 65-84. 
sagen, dass in diesen Gattungen die Entdeckung der psychischen Dimensionen, des Unbewussten, am ehesten vonstattengehen konnte.

Sterne betont die Doppeldeutigkeit bzw. Uneindeutigkeit der Dinge auf allen Ebenen. Ein Verfahren, das Karikatur und Satire gleichermaßen verwenden, ist das uralte der sprechenden Namen. Wenn schon Hogarth in $A$ Rake's Progress den Protagonisten seiner Serie Tom Rakewell nennt, dann bedeutete der Nachname für den Vater des Liederlichen, dass er durch ausgeprägten Geiz seinen Besitz „gut zusammengeharkt" hat („to rake well“), für den Sohn dagegen, der das väterliche Erbe hemmungslos verprasst, dass er ein ausgeprägter „rake“, ein liederlicher Lebemann ist. ${ }^{61}$ Auch das Hogarth'sche Verfahren, durch Bilder an der Wand das eigentliche Geschehen zu kommentie$\mathrm{ren}^{62}$, nutzt die indirekte Redeweise und fordert den Anteil des Betrachters ein. Wenn Tristram zum Entsetzen des Vaters den falschen Namen erhält, dann weist uns Sterne schon damit darauf hin, dass Namen Bedeutung tragen, nur keine offensichtliche. Auch Namen verweisen, „nomen est omen“. Doch Sterne ist auch bei der Anwendung dieses Verfahrens nicht eindeutig, erneut sind unsere Assoziation und Projektion gefordert, und erneut können wir nicht sicher sein, ob wir nicht überziehen.

Die Sterne-Forschung ist auf die Kunstnamen besonders in latinisierter Form aufmerksam geworden, denn diese Namen sind schon selbst aufgrund von Verschiebung, von Übersetzung, entstanden. ${ }^{63}$ So fällt in der höchst überraschend im 20. Kapitel des dritten Buches eingeschobenen „Vorrede des Verfassers" - er hat plötzlich Zeit, sie zu verfassen, da alle Protagonisten entweder eingeschlafen oder wie Dr. Slop mit dem Geburtsvorgang beschäftigt sind - eine Reihe lateinischer Namen, die entweder auf real existierende historische Größen oder aber auf gänzlich erfundene Personen bezogen sind.$^{64}$ Es geht um die Lockesche Unterscheidung von Witz und Urteil, die Lockes Meinung nach einander ausschließen. Dem stimmt auch ein Phutatorius zu, was bewusst paradox ist, denn der Name verweist auf das lateinische fututor, einen den Geschlechtsakt Vollziehenden. ${ }^{65}$ Verstehen wir Sterne richtig, so will er uns sagen, dass Phutatorius genau das Gegenteil von dem meint, was er sagt. Er ist nicht Anhänger der Locke'schen Dominanz des Urteils, sondern wie

${ }^{61}$ Ronald Paulson, Hogarth's Graphic Works, 3. überarb. Ausgabe, London, 1989, Kat.Nr. 132, S. $91 \mathrm{f}$.

62 Zur Herleitung des Verfahrens aus der Bologneser Grafik des 17. und 18. Jahrhunderts: Hilde Kurz, ,Italian Models of Hogarth's Picture Stories“, in: Journal of the Warburg and Courtauld Institutes 15 (1952), S. 136-168.

63 Neben Melvyn News Kommentar zu Tristram Shandy: Laurence Sterne, The Life and Opinions of Tristram Shandy, Gentleman, The Notes, hrsg. v. Melvyn New (The Florida Edition of the Works of Laurence Sterne, Bd. 3), Gainesville (Fla.) u. a., 1984, siehe Elizabeth M. Wendell, Der Leser als Protagonist. Didaktische Strukturen in Laurence Sternes „, Tristram Shandy“, Frankfurt am Main/Bern/Las Vegas, 1979, S. 41-47. 
Sterne Anhänger des Witzes - und mit dem Witz verbindet Sterne Natur und Trieb. Wie wir später erfahren, ist auch Phutatorius triebbestimmt.

Unmittelbar vor dem Kapitel zu Phutatorius, im 26. Kapitel des vierten Buches, hat ein Dr. Kysarcius sein Vorkommen, von dem es nun wieder verräterisch heißt: „[E]r war von den Kysarcii aus den Niederlanden ${ }^{\text {“66. }}$. Offenbar wird damit wieder auf eine Neigung angespielt, denn gelesen ergibt sich aus dem Namen kiss-arse, und die feine lateinische Form wird umgangssprachlich konterkariert. Die Spannung liegt also im Namen selbst. Wenn der kluge Kommentar der Florida-Ausgabe das Schachtelwort Kysarcius höchst fein als eine mögliche Sterne'sche Übersetzung von baise $\mathrm{cul}^{l^{67}}$ deklariert, dann möchten wir dem Kommentator wünschen, dass er darin eine Anspielung auf den Wortspieler Marcel Duchamp verborgen hat, der seiner Mona Lisa die Beischrift L. H. O. O. Q. (,Elle a chaud au cul“- „Sie ist heiß am Hintern“, gemeint: Sie ist läufig) gegeben hat. ${ }^{68} \mathrm{Zu}$ überlegen wäre, aber dies müsste Herbert Molderings entscheiden, ob Duchamps Wortspiel sich nicht gleichermaßen Sterne und Goya verdankt.

Etwas anders verhält es sich mit dem Helden von Sternes Text, mit Tristram selbst. Er, der den falschen Namen bekommen hat, soll ihn unabsichtlich bekommen haben? Sein Name soll nicht sprechend sein? Man vermag es kaum zu glauben. Der Name Tristram, selbst wenn er keltischen Ur-

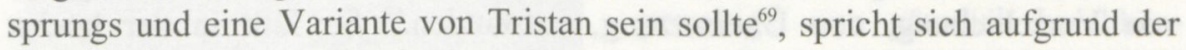
Ballung der Konsonanten in der Mitte nur schwer aus. Die Hälfte der Menschheit glaubt ohnehin, er hieße „Tristam“, das spricht sich in der Tat leichter. Tristram kann man nur aussprechen, wenn man mit leichtem Stocken den Namen in zwei Silben ausspricht: „Trist-ram“. Im 36. Kapitel des fünften Buches reflektiert Walter vor dem staunenden Toby thesenhaft über die Radikalfeuchtigkeit und die unnatürliche Trockenheit (auch) der menschlichen Körper. Und bei der Erörterung des Verhältnisses von Hitzigkeit und Feuchtigkeit, von Feuer und Wasser, kommt ihm Aristoteles' Bemerkung in den Sinn: "Quod omne animal post coitum est triste“, die Bemerkung zur automatischen Traurigkeit nach dem Geschlechtsakt, wo Hitzigkeit und Feuchtigkeit gegangen sind. ${ }^{70}$ Der arme Tristram, dem seine Kastration ins Gesicht geschrieben

${ }^{66}$ Sterne, s. Anm. 4, S. 338-340, Zitat S. 338 (26. Kap., 4. Buch).

67 Sterne, s. Anm. 63, S. 239 f.

${ }^{68}$ Herbert Molderings, „Vom Tafelbild zur Objektkunst. Kritik der ,reinen Malerei““, in: Werner Busch und Peter Schmoock (Hrsg.), Kunst. Die Geschichte ihrer Funktionen, Weinheim/Berlin, 1987, S. 231.

69 Zum Namen „Tristram“ siehe http://www.meaning-of-names.com/names/t-names-44.asp; http://en.wikipedia.org/wiki/tristan_(name) (17. Oktober 2011); Patrick Hanks und Flavia Hodges, A Dictionary of First Names, Oxford, 1990, unter „Tristram“, S. 323.

70 Sterne, s. Anm. 4, S. 424 (36. Kap., 5. Buch). Aristoteles, Fünf Bücher von der Zeugung und Entwicklung der Tiere, übers. u. erl. v. Hermann Aubert und Friedrich Wimmer (Aristoteles, Werke, Bd. 3), Neudruck der Ausgabe Leipzig, 1860, Aalen, 1978, Erstes Buch, 725b, S. 93 : „Aber bei den meisten [Tieren] und im Allgemeinen erfolgt nach der Ausübung der Wollust aus der angegebenen Ursache vielmehr Erschlaffung und Kraftlosigkeit.“ 
steht, ist in der Tat eine traurige Erscheinung, ein neuer Ritter der traurigen Gestalt. Und ein „Ram“ ist ein Rammbock, so dass es sich bei Tristram wahrlich um einen höchst traurigen Rammbock handelt, der seiner natürlichen $\mathrm{Be}$ stimmung nie nachkommen wird. Er ist keiner der sprichwörtlich triebgeleiteten Schaf- oder Ziegenböcke, wie Rembrandt sie neben seinen „Eulenspie-

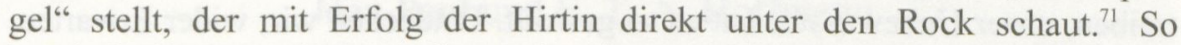
komisch die Sterne'sche Satire auch sein mag, sie ist zudem traurig und melancholisch, so komisch, traurig und melancholisch wie der Autor selbst, der bekanntlich auch Tristram und Yorick zugleich ist.

Nun mag man eine derartige Namenslektüre für weit oder auch zu weit hergeholt halten. Doch Sterne gibt uns, wie so oft, an ganz anderer Stelle einen ziemlich direkten Hinweis, und es verwundert, dass die Forschung ihn nicht aufgegriffen hat. Im Tagebuch für Eliza heißt es zum 24. April:

„Und jetzt muss ich Dir, wenn ich genug Kraft und Schwung habe, meine Feder bis ans Ende der Seite zu führen, eine drollige Geschichte [, whimsical a Story'] erzählen, die so tragikomisch [,comically dis-astrous'] ist, wie sie sich nur jemals in unserer Familie ereignet hat - Shandys Nase, sein Name, sein Fallfenster sind nichts dagegen." ${ }^{672}$

Die nun folgende Geschichte kreist um die bei Laurence Sterne vermutete Geschlechtskrankheit. Tristrams Name ist durch zwei Geschichten eingerahmt: durch die Geschichte von Shandys Nase, die das Opfer von Dr. Slops Geburtszange wurde und damit, durch die permanente Penisallusion aller im Buch vorkommenden Nasen, ihrem armen Träger die Impotenz ins Gesicht schreibt, und vor allem durch die Geschichte des Fallfensters, das beim Heruntersausen den bedauernswerten, aus dem Fenster pinkelnden Knaben noch einmal verstümmelt und um jede Möglichkeit des geschlechtlichen Vollzuges bringt. Schon diese Rahmung verweist darauf, dass der Name Tristram bewusst gewählt wurde. Tristrams ganzes Leben ist von diesem Hauptmanko überschattet. Der Traurigkeit ist kein Ende.

Sternes Zwiegespräch mit dem Leser weist ihn noch und noch auf die Unmöglichkeit einer erfüllten Kommunikation hin, Eindeutigkeit und logische Abfolge sind nicht zu erreichen. Was beim Leser jedoch ausgelöst wird, ist ein Bewusstsein seines eigenen Bedürfnisses nach gelingender Kommunikation, nach einer Adressierung der eigenen Bedürfnisse an den anderen. Er möchte nichts anderes als lieben und geliebt werden. In der Realität kommt dieses Bedürfnis nicht zur Erfüllung. So bleiben Sterne die sprachlichen Metaphern als Ausdruck einer Umschreibung des Gewünschten, wir sollen es ahnen, es auch als unseren Wunsch erkennen - und sei er noch so triebhaft. In den beschrie-

71 Ausst.-Kat. Rembrandt the Printmaker, hrsg. v. Erik Hinterding, Ger Luijten und Martin Royalton-Kisch, The British Museum, London, 2000, Kat.-Nr. 46, S. 200-203.

72 Laurence Sterne, Tagebuch für Eliza - Empfindsame Reise, hrsg. v. Lutz Zimmermann, Frankfurt am Main/Berlin, 1989, S. 15 (24. April 1767); engl. Ausgabe in: Laurence Sterne, A Sentimental Journey Through France and Italy, Ware, 1995, Zitate S. 106. 
benen Posen jedoch hält Sterne das Geschehen an, zwingt uns, über ihre Sinnfälligkeit nachzudenken, zu grübeln darüber, was der Autor von uns will. Wir lassen die bis dahin erzählte Geschichte Revue passieren, wir rufen, auch gerade durch die sprachliche Wiederholung, die Pose vor unser geistiges Auge: assoziieren, imaginieren, phantasieren, lassen uns in ganz andere Bereiche treiben, unser Unbewusstes hat es so gewollt. Entdecken wir, wider Erwarten, das Vorbild der bildhaft markierten Pose, dann kommt unsere Assoziationsmaschinerie erst eigentlich in Gang, wir wollen geradezu eine Ähnlichkeit zwischen Vorbild und Nachbild herbeizwingen. Wir wollen die Lücke zwischen Vor- und Nachbild schließen, werden uns unserer Bemühungen bewusst, setzen schließlich Bedeutungen, wohl wissend, dass sie nur von uns stammen und nicht zwingend sind. Sinnstiftung erweist sich als ein ambivalentes Geschäft. Misslungene, aber notwendig immer erneut versuchte Kommunikation ist unser Schicksal - das haben Virginia Woolf und James Joyce, aber auch Thomas Mann und Arno Schmidt und nicht zuletzt Samuel Beckett von Sterne gelernt. 